\title{
Outage prediction for ultra-reliable low-latency communications in fast fading channels
}

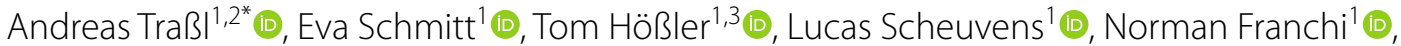 \\ Nick Schwarzenberg ${ }^{1}$ (ID) and Gerhard Fettweis ${ }^{1,2,3}$ (1)
}

*Correspondence: andreas.trassl@tu-dresden.de ${ }^{1}$ Vodafone Chair Mobile Communications Systems, Technische Universität Dresden, Dresden, Germany Full list of author information is available at the end of the article

\begin{abstract}
The addition of redundancy is a promising solution to achieve a certain quality of service (QoS) for ultra-reliable low-latency communications (URLLC) in challenging fast fading scenarios. However, adding more and more redundancy to the transmission results in severely increased radio resource consumption. Monitoring and prediction of fast fading channels can serve as the foundation of advanced scheduling. By choosing suitable resources for transmission, the resource consumption is reduced while maintaining the QoS. In this article, we present outage prediction approaches for Rayleigh and Rician fading channels. Appropriate performance metrics are introduced to show the suitability for URLLC radio resource scheduling. Outage prediction in the Rayleigh fading case can be achieved by adding a threshold comparison to state-of-the-art fading prediction approaches. A line-of-sight (LOS) component estimator is introduced that enables outage prediction in LOS scenarios. Extensive simulations have shown that under realistic conditions, effective outage probabilities of $10^{-5}$ can be achieved while reaching up-state prediction probabilities of more than $90 \%$. We show that the predictor can be tuned to satisfy the desired trade-off between prediction reliability and utilizability of the link. This enables our predictor to be used in future scheduling strategies, which achieve the challenging QoS of URLLC with fewer required redundancy.
\end{abstract}

Keywords: Channel prediction, URLLC, Radio resource scheduling

\section{Introduction}

One of the main pillars of the fifth generation (5G) mobile broadband standards is ultrareliable low-latency communications (URLLC), which aims to provide extremely high service availabilities paired with latency values of only a few milliseconds. To realize even more ambitious quality of service (QoS) requirements compared to 5G, URLLC inevitably has to play a key role also during research of the sixth generation (6G) mobile broadband standards [1, 2].

The ongoing development of URLLC is driven by a wide variety of applications. In recent years, many of these applications were industry-focused, where wireless author(s) and the source, provide a link to the Creative Commons licence, and indicate if changes were made. The images or other third party material in this article are included in the article's Creative Commons licence, unless indicated otherwise in a credit line to the material. If material is not included in the article's Creative Commons licence and your intended use is not permitted by statutory regulation or exceeds the permitted use, you will need to obtain permission directly from the copyright holder. To view a copy of this licence, visit http:// creativecommons.org/licenses/by/4.0/. 
solutions allow for shorter product cycles, more product individualization and an overall increased flexibility [3]. One major challenge is wireless closed-loop control as losing packets and the latency of the transmission might lead to plant instability, which in turn could cause damage or even human harm. Latency-critical mobile connectivity is also required when humans are involved in the control loop $[4,5]$. In industry, this is the case, e.g., in teleoperating applications or during installation of machines, where a human could train the machine instead of programming it. In the future, URLLC might also find its way to consumer products for entertainment, for health or even for human learning.

The major challenges for URLLC are the imperfections of the wireless link, especially the fast fading of the channel. Due to reflections in the environment, many copies of the transmit signal arrive at the receiver simultaneously and interfere which each other. When the transmitter or the receiver moves, the channel conditions continuously change since the waves interfere differently at different locations [6]. In the best case, all signals constructively add up at the receive antenna. In the worst case, however, all signals destructively cancel each other out, effectively leading to zero receive power. Situations where the receive power is low, so-called outages, have to be avoided for the successful realization of URLLC.

Different from well-established conventional approaches that cost severe resources either in hardware (spatial diversity through many antennas and signal processing chains) or at the air interface (bandwidth), the basic concept of this article is to monitor the fast fading channel and schedule users only to resources that are operational. Due to the spatial variation of the channel, a resource that is in outage for one user can have perfect channel conditions for another. Therefore, this approach is expected to realize URLLC's ambitious QoS targets while keeping the required additional resource consumption low. This is of utmost importance when considering the scalability of a URLLC deployment, i.e., when many devices need to be served simultaneously. The resource consumption becomes even more important when realizing high payload applications with latency requirements, e.g., cloud rendered virtual or augmented reality.

Rapidly varying channel conditions are challenging when monitoring fast fading channels. In a real deployment, a monitoring delay $\tau$ exists between receiving the last channel observation, scheduling and eventually transmitting the actual payload. Consider the case that users were scheduled based on the last channel observation. In this case, many actual outages would be missed; i.e., the channel is monitored to be operable at time $t$, but non-operable during payload transmission at $t+\tau$. The performance for Rayleigh fading is visualized in Fig. 1, where the effective outage probability of such a system $\operatorname{Pr}$ (effective outage) is plotted against the Doppler frequency normalized monitoring delay $\tau f_{\mathrm{m}}$ for different fading margins $F$. It is clearly visible that most of the monitoring gain disappears already for small monitoring delays. In other words, with increasing delay the monitoring quickly becomes pointless and the effective outage probability asymptotically converges towards the average link outage probability.

A solution to overcome this time delay is to employ predictive methods. Although the fast fading channel conditions change quickly, they still change continuously, which enables predictions. The main contribution of this article is to describe the design and the 


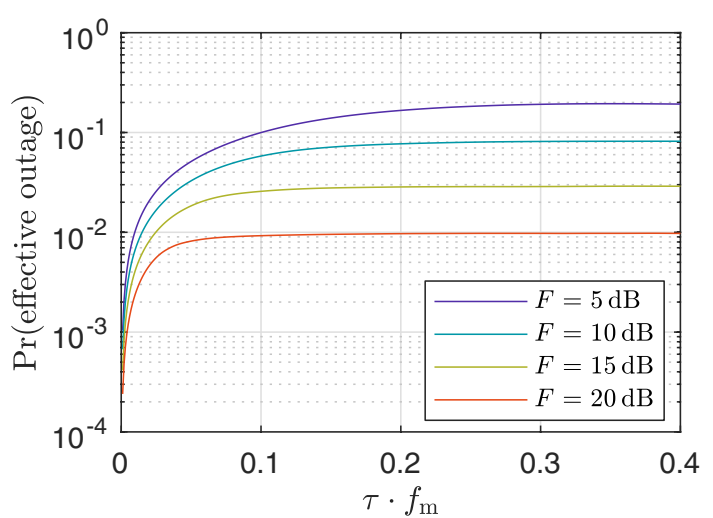

Fig. 1 Performance of outage identification, when relying purely on the latest channel observation

performance of an outage predictor. This article is based on and extends our previous works $[7,8]$, where outage predictors for Rayleigh and Rician fading were proposed for the first time. The contributions of this article are summarized as follows:

- Outage predictors for Rayleigh and Rician fading channels are described.

- The Rayleigh und Rician fading outage predictors are compared. Their differences are highlighted and their areas of application are contrasted.

- Relevant related work regarding fading prediction is discussed.

- An analysis of the predictor parameters for Rician fading is presented. The parameters define the number and periodicity of channel observations at the input of the predictor.

- Performance metrics tailored to the use-case of radio resource scheduling for URLLC are proposed.

- Performance evaluation is conducted by means of extensive simulation. Compared to our previous works $[7,8]$, generalized results are provided.

\section{Methods/experimental}

This article aims to study the performance of a novel outage prediction scheme in Rayleigh and Rician fading channels. The predictor combines a Wiener filter with a threshold comparison for the identification of future outages. In the Rician fading case, additional line-of-sight (LOS) parameter estimation is employed. The focus during design and analysis of the prediction scheme is set on a prospective application to URLLC radio resource scheduling.

The performance of the outage prediction scheme is analyzed using analytical statements and Monte-Carlo computer simulation for a practical set of parameters. Noisy channel coefficients are generated randomly and fed into the predictor. The predictions are then compared with the respective true future value. Evaluation is conducted using classical binary classification analysis and application-related metrics that are proposed in this work. The number of repetitions is individually specified during discussion. The 
underlying data of this study is generated from mathematical models, which are completely described in Sect. 4.

\section{Related work}

Estimating the current state and predicting future behavior of wireless channels has been a research challenge for many years. As expected, the used estimation methods have been evolving along with the wireless communications technologies and standards.

With the ever-growing demand of higher transmission rates without sacrificing transmission quality in terms of bit error rate (BER), the availability of channel state information became a necessity. Adaptive transmission techniques allow a more efficient resource usage, e.g., by choosing the modulation scheme according to the current fading conditions [9]. Early approaches were based on the sum-of-sinusoids (SOS) modeling. The deterministic fading modeling is based on the estimation of Doppler shifts, amplitudes and phases of the overlaid sinusoids through ESPRIT- and MUSIC-based algorithms $[10,11]$. This is done under the assumption that scatterers in the environment remain constant and the process is dominated by a few dominant scatterers. The first advances were followed by many variants of auto-regressive (AR) approaches. These predicted channel samples as a weighted sum of previous samples using the minimum mean square error (MMSE) criterion. This method requires an estimate of the correlation function of the channel samples. Promising results were obtained, e.g. in [9], where a Wiener filter is used for the prediction of in-phase and quadrature (I/Q) Rayleigh fading channels. The predictor is tested using data generated by ray-tracing and real data from vehicular channel measurements. Similar investigations were performed in [12, 13]. In these works, an unbiased power predictor is additionally derived based on solely the channel power instead of the complex channel gain. The works $[9,14,15]$ extended the predictor by the use of efficient adaptive filtering techniques to update the predictor coefficients in case of varying long-term channel conditions. Motivation for investigating fading prediction techniques was exclusively the raise of spectral efficiency.

When orthogonal frequency-division multiplexing (OFDM) became part of the fourth generation (4G) mobile broadband standard, channel prediction research was directed towards adaptive bit allocation in OFDM symbols, aiming again to increase the spectral efficiency. In [16], the MMSE predictor, the Wiener filter predictor, as well as the adaptive methods normalized least mean squares (NLMS) and recursive least squares (RLS) are derived for a single input single output (SISO) OFDM system. The predictors are compared in terms of their mean prediction errors. Similarly, for SISO OFDM systems, a simplified MMSE predictor and its extension to the adaptive least mean squares (LMS) and RLS techniques were proposed in [14]. Evaluations were conducted in terms of average prediction error and spectral efficiency. Similar to OFDM, [15] also exploits channel prediction for adaptive frequency hopping by adapting transmission parameters to the channel conditions at the next chosen frequency. Other approaches include the estimation of time varying fading parameters with the help of Kalman filter variants to enhance the prediction performance [17]. In [18], a Kalman filter is used to directly predict Rayleigh fading. The used state space model is based on the SOS model. Recently, opposing to statistical methods, machine learning approaches have been proposed for fading channel prediction. For example, in [19] a back propagation neural network is used to 
predict I/Q channel coefficients in Rayleigh fading. A recurrent neural-network-based approach is used in [20] and analyzed in terms of average prediction errors and bit error rates. Those data-driven approaches do not require any modeling or parameterization.

In today's $5 \mathrm{G}$ and future's 6G mobile broadband standard, very high data rates are only one of the envisioned features. For the realization of URLLC, more attention needs to be directed towards the reliability of transmissions rather than solely maximizing spectral efficiency. Consequently, research on fast fading channel prediction has to perform a paradigm shift as well. To allow the scheduling system to achieve a certain QoS, the predictor needs to be built around suitable reliability measures. For URLLC, the analysis of average prediction errors and BERs is not enough anymore. Under the URLLC premise, only very few investigations have been conducted. In [21, 22] cooperative communications schemes, where messages are transmitted over multiple relays, are investigated for URLLC. The authors employ fading monitoring and prediction to choose the most suitable relays. It is shown that the coherence time is an insufficient metric to quantify the reliability of fading prediction methods. This article contributes to fill this gap and provides prediction methods and metrics for general URLLC architectures.

\section{System model}

The overall system model considered in this article is shown in Fig. 2. It is assumed that the user equipments (UEs) periodically transmit training signals, which are used to acquire channel information between the base station (BS) and the individual UE. We assume channel reciprocity such that by measuring the uplink (UL) channel, the downlink (DL) channel can also be rated. This is practical after a calibration phase to account for differences in the circuits of transmitter and receiver as shown in [23]. Monitoring the uplink channel is preferred over the downlink since thereby the necessary information is directly available to the scheduler at the BS. The channel estimations are fed into the outage predictor, whose design and performance will be the main topic of this article. For each monitored carrier frequency, the predictor calculates for the next possible UL and DL scheduling opportunity if the respective link is operational or not. Based on this information, the scheduler allocates resources to the UEs. As usual, the scheduling decision is transmitted to the UEs. In the following, the necessary assumptions for the fading channel and the communications system are explained.

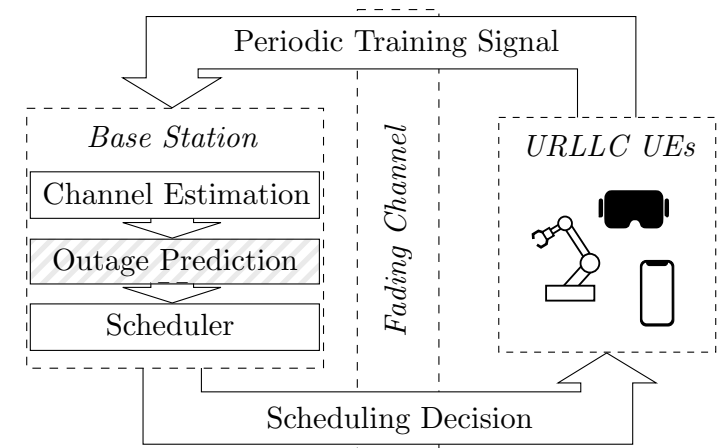

Fig. 2 Overall system model. The outage predictor is highlighted and topic of this article 


\subsection{Fading channel}

In this article, we first consider Rayleigh fading with classical Doppler spectra before extending our findings to the Rician fading model. Rayleigh fading can be used to model challenging non-line-of-sight (NLOS) conditions and is often used as a starting point for analysis due to its beneficial mathematical properties. In the second part of this paper, we extend our findings to the Rician fading case by allowing for a LOS component. By doing so the question how the presence of a LOS component affects the prediction performance is answered.

\subsubsection{Rayleigh fading}

Rayleigh fading assumes that numerous independent multi-path components arrive at the receive antenna simultaneously. In this case, the central limit theorem can be applied and therefore the real and imaginary part of the channel coefficient $h(t)$ can be modeled as Gaussian distributed. Thus, the channel coefficient

$$
h(t)=\sqrt{2} \sigma \cdot h_{\mathrm{NLOS}}(t),
$$

follows a zero mean complex Gaussian distribution with variance $2 \sigma^{2}$, since we define $h_{\mathrm{NLOS}}(t) \sim \mathcal{C N}(0,1)$. The variance of the complex channel coefficient $2 \sigma^{2}$ is then determined by the mean power of the channel $\Omega_{\mathrm{NLOS}}$ according to $2 \sigma^{2}=\Omega_{\mathrm{NLOS}}$.

An underlying assumption for the widely assumed classical Doppler spectrum is that waves arrive solely in the horizontal plane with equally distributed angles of arrival. The UE is considered to move with a constant velocity $v$ in an otherwise static environment, which results in a maximum Doppler shift $f_{\mathrm{m}}$. It is well-known that this leads to the classical Doppler spectrum with its autocovariance function

$$
r_{\mathrm{NLOS}}\left(t_{1}, t_{2}\right)=2 \sigma^{2} J_{0}\left(2 \pi f_{\mathrm{m}}\left(t_{2}-t_{1}\right)\right) .
$$

Thereby, $J_{0}$ denotes the zeroth order Bessel function of the first kind.

\subsubsection{Rician fading}

When additionally allowing for a LOS component, the Rician fading case arises with its I/Q channel coefficient [24]

$$
h(t)=\sqrt{2} \sigma \cdot h_{\mathrm{NLOS}}(t)+A \cdot h_{\mathrm{LOS}}(t) .
$$

The NLOS component $\sqrt{2} \sigma \cdot h_{\mathrm{NLOS}}(t)$ is similar to the Rayleigh fading case described above. The LOS component $A \cdot h_{\mathrm{LOS}}(t)$ is modeled to be purely deterministic following

$$
A \cdot h_{\mathrm{LOS}}(t)=A \cdot \exp \left(\mathrm{j}\left(2 \pi f_{\mathrm{D}, \mathrm{LOS}}\left(t-t_{0}\right)+\varphi_{0}\right)\right) .
$$

In this formula, $A$ is the amplitude, $f_{\mathrm{D}, \mathrm{LOS}}$ is the Doppler frequency, $\varphi_{0}$ is the initial phase of the LOS component and $t_{0}$ is the reference time at which the phase of the LOS component equals the initial phase $\varphi_{0}$.

In Rician fading the $K$-factor defines the ratio of power in the LOS component $\Omega_{\mathrm{LOS}}$ over the power in the NLOS component $\Omega_{\mathrm{NLOS}}$ 


$$
K=\frac{\Omega_{\mathrm{LOS}}}{\Omega_{\mathrm{NLOS}}}=\frac{A^{2}}{2 \sigma^{2}}
$$

Thus, the standard deviation of the complex NLOS component $\sqrt{2} \sigma$ and the amplitude of the LOS component $A$ can alternatively be expressed over the $K$-factor and the average power $\Omega=\Omega_{\mathrm{LOS}}+\Omega_{\mathrm{NLOS}}$ using

$$
\sqrt{2} \sigma=\sqrt{\frac{\Omega}{K+1}}, \quad A=\sqrt{\frac{\Omega K}{K+1}} .
$$

For the special case of $K=0$, (1) and (3) coincide. Thus, the more general Rician fading model also includes the Rayleigh fading case.

\subsubsection{Two state fading model}

Our predictor is built upon an abstract fading model in which the fading is classified in two states up and outage depending on the channel gain. This fading model is depicted in Fig. 3 . The respective fading state is based on the relation of the channel gain to a chosen threshold value $\left|h_{\min }\right|$. A different form to characterize the threshold $\left|h_{\min }\right|$ is the fading margin $F=\left|h_{\text {avg }}\right|^{2} /\left|h_{\min }\right|^{2}$, which relates the threshold $\left|h_{\min }\right|$ to the average channel gain $\left|h_{\text {avg }}\right|$. When the channel gain is greater than the threshold $|h(t)|>\left|h_{\min }\right|$ the current fading state is denoted as up. In the up-state the signal/noise ratio (SNR) at the receiver is high enough for an URLLC application to be working satisfactory. Packet errors are still possible in the up-state, but the probability for an error is low and long error bursts are rare. Analogously, an outage occurs if the channel gain is below the threshold value $|h(t)|<\left|h_{\min }\right|$. In outage, the SNR is usually too low for successful decoding, leading to high probabilities of packet errors and long error bursts. Following these considerations, a URLLC service is expected to work satisfactory in the up-state and fail in the outage state.

\subsection{Communications system and channel estimation}

For the communications system we assume that the transmission between the UE and the BS is affected only by the fading of the wireless channel, resulting in the complex channel coefficient $h(t)$, and complex white Gaussian noise (CWGN) $n(t)$ with variance $2 \sigma_{n}^{2}$. Hence, the transmit signal $x(t)$ and the receive signal $y(t)$ are related by

$$
y(t)=x(t) \cdot h(t)+n(t) .
$$

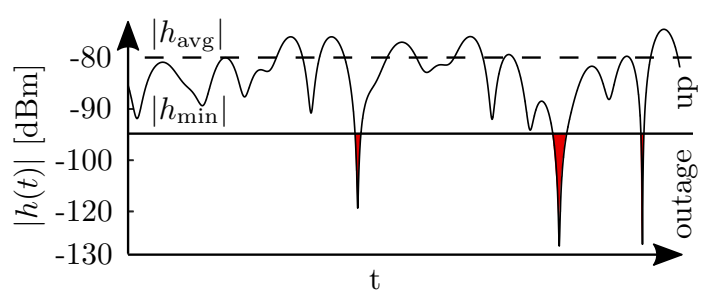

Fig. 3 Two state fading model 
To acquire information about the wireless channel, a column vector $\boldsymbol{p}$ consisting of $P$ pilot symbols is transmitted for the estimation of $h(t)$. Thus, when taking (7) into account, this leads to

$$
\boldsymbol{y}=\boldsymbol{p} \cdot h(t)+\boldsymbol{n} .
$$

With knowledge of the sent pilot symbols at the receiver, the influence of the fading can be observed by estimating the complex channel coefficient $h(t)$. For this purpose, we use the minimum variance unbiased (MVU) channel estimator [25]

$$
\hat{h}(t)=\left(\boldsymbol{p}^{H} \boldsymbol{p}\right)^{-1} \boldsymbol{p}^{H} \boldsymbol{y},
$$

which corresponds the least squares (LS) and maximum likelihood (ML) channel estimator. Inserting (8) in (9) yields

$$
\hat{h}(t)=h(t)+n^{\prime}(t) .
$$

Thus, under the given assumptions the estimate $\hat{h}(t)$ is superimposed by CWGN $n^{\prime}$ with variance $2 \sigma_{n^{\prime}}^{2}=2 \sigma_{n}^{2}\left(\boldsymbol{p}^{H} \boldsymbol{p}\right)^{-1}$. When only one pilot is used for channel estimation, $2 \sigma_{\mathrm{n}}^{\prime 2}=2 \sigma_{\mathrm{n}}^{2}$ applies. The relationship between channel estimation and noise (10) is the starting point for the derivation of the predictor. The performance of the predictor will be determined by the SNR of the channel estimation, $\mathrm{SNR}=\frac{\Omega}{2 \sigma_{\mathrm{n}}^{\prime 2}}$.

\section{Problem statement}

Prediction of the channel state involves so-called binary classification. Usually, the results of the classification problem are simply called positive and negative. Since the outage predictor aims at predicting outages, this article defines the up-state prediction as the negative and the outage prediction as the positive classification result. In binary classification, four potential outcomes exist. Apart from true positive and true negative (correct classification), also the two error types false positive and false negative prevail. In the context of outage prediction these four outcomes represent the following:

- true positive-detection of a future outage

- true negative-detection that an outage will not occur

- false positive-miss that an outage will not occur

- false negative-miss of a future outage

The evaluation of such binary classification problems is well known and various metrics for different applications are available. Three important metrics are summarized from [26], where a good overview is given. An intuitive metric for evaluation of a classifier is its accuracy, which is defined as

$$
\text { accuracy }=\frac{\mathrm{TP}+\mathrm{TN}}{\mathrm{TP}+\mathrm{FP}+\mathrm{TN}+\mathrm{FN}} .
$$

In this formula TP is the number of true positives and TN is the number of true negatives. Similarly, FP is the number of false positives and FN is the number of false negatives. An accuracy of 0.8 means that $20 \%$ of the prediction results are wrong. However, 
by investigating the accuracy metric alone it is impossible to say if these errors are false positives or false negatives. The metric is of limited benefit for URLLC outage prediction since the error types have a different impact on the reliability of the wireless link. In the context of QoS-focused URLLC, false negative classifications have a much higher impact than false positives as they can be a cause for transmission errors which ultimately lowers the reliability of the wireless communications system. This emphasizes the importance of choosing the right metric. Investigating the individual probabilities for correct or wrong classification allows for more explicit statements. Two common metrics to fully describe the classifier are the probability of false alarm (also known as the false positive rate)

$$
\operatorname{Pr}(\text { false alarm })=\frac{F P}{T N+F P}
$$

and the probability of detection (also known as the true positive rate)

$$
\operatorname{Pr}(\text { detection })=\frac{\mathrm{TP}}{\mathrm{FN}+\mathrm{TP}} .
$$

The above metrics are well suited to investigate binary test results. However, they do not provide intuitive interpretation in the context of URLLC radio resource scheduling. For example, in the case of very high Rician $K$-factors even $\operatorname{Pr}$ (detection) $=0$ (all outages are missed) could be acceptable as outages are already very rare. Therefore, $\operatorname{Pr}$ (detection) has only little qualitative meaning if the predictor performs well enough for scheduling purposes. Here, we propose two new metrics: the compound probability for an up-state prediction, but the channel being truly in outage

$$
\operatorname{Pr}(\text { effective outage })=\frac{\mathrm{FN}}{\mathrm{TP}+\mathrm{FP}+\mathrm{TN}+\mathrm{FN}}
$$

and the average probability to have an up-state prediction on the monitored link

$$
\operatorname{Pr}(\text { predicted up })=\frac{\mathrm{FN}+\mathrm{TN}}{\mathrm{TP}+\mathrm{FP}+\mathrm{TN}+\mathrm{FN}} .
$$

First, $\operatorname{Pr}$ (effective outage) covers the risk of fatal failures due to prediction errors. Thus, this metric enables statements about the reliability of the system. For example, $\operatorname{Pr}($ effective outage $)=10^{-3}$ indicates that on average 1 in 1000 predictions will result in an outage. Here, we assume that a (perfect) scheduler can prevent any predicted outage, since the design of the scheduler is beyond the scope of this article. Second, $\operatorname{Pr}$ (predicted up) is defined in a way to assess the utilizability of the resource, i.e., how often the observed link can be used for URLLC traffic of a specific UE. The more false alarms occur, the lower $\operatorname{Pr}$ (predicted up) gets. For example, $\operatorname{Pr}$ (predicted up) $=0.8$ indicates that the observed link can be considered for URLLC traffic in $80 \%$ of the time, whereas in the remaining $20 \%$ the link will not be assigned to that particular UE for transmission.

The ultimate goal for the predictor is to maximize $\operatorname{Pr}$ (predicted up) and minimize $\operatorname{Pr}$ (effective outage) given a certain prediction horizon $t_{p}$. 


\section{Outage prediction}

In the following, we describe the structure of the outage predictor. We first address the Rayleigh fading case and pursue to the more complex Rician fading afterwards.

\subsection{Rayleigh fading prediction}

The outage predictor for the Rayleigh fading case is shown in Fig. 4 and its structure is briefly explained here before providing mathematical details.

The starting point for outage prediction is a history of channel estimations which is collected at the input of the outage predictor. Afterwards, I/Q channel coefficients need to be predicted from the available channel estimations by an appropriate prediction technique. In order to obtain a binary prediction for the channel state (up or outage), the predicted I/Q channel coefficient is compared with a threshold $\left|h_{\min }^{\prime}\right|$. Subsequently, an outage prediction is available which can be used, e.g., for scheduling purposes. For the Rayleigh fading case, the exact distribution of the I/Q prediction error is known at the Wiener filter output. Thus, additionally to the outage prediction also the probability for a future outage can be calculated for future time instants. In the next sections, a detailed description of each block in Fig. 4 is provided.

\subsubsection{I/Q prediction}

Based on the history of channel estimations, predictions of I/Q channel coefficients need to be calculated. For this purpose, a well investigated Wiener-filter-based approach is employed $[9,12]$. It was shown that the Wiener filter has a promising performance not only under the Rayleigh fading assumption, but also for real fading channels where empirical covariances need to be utilized. In contrast to machine learning-based approaches, analytical statements about the prediction error can be derived, which allow calculation of future outage probabilities in the Rayleigh fading case. For these reasons, the Wiener filter was preferred over other available fading prediction techniques. Nevertheless, if only the outage prediction is of interest, other I/Q prediction techniques can be easily incorporated into the proposed framework as well and replace the Wiener filter. The key statements to implement the Wiener filter for the Rayleigh case are summarized from [12].

For a prediction horizon $t_{\mathrm{p}}$, the prediction of the I/Q channel coefficient

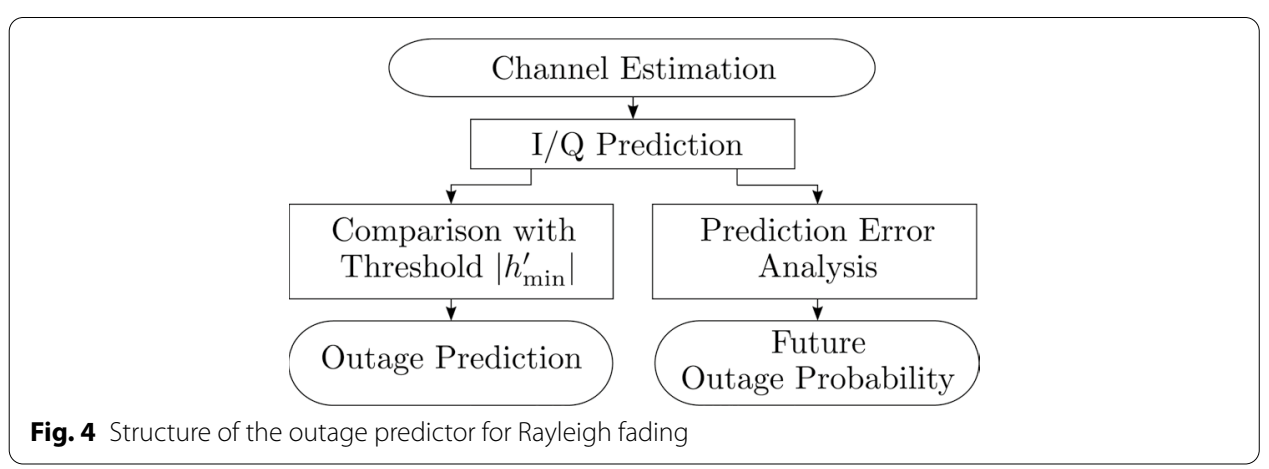




$$
\hat{h}\left(t+t_{\mathrm{p}} \mid t\right)=\boldsymbol{\varphi \theta}
$$

is the output of a finite impulse response (FIR) filter with coefficients $\boldsymbol{\theta}$. The observation vector

$$
\boldsymbol{\varphi}=\left[\begin{array}{llll}
\hat{h}(t) & \hat{h}(t-\Delta t) & \ldots & \hat{h}(t-(M-1) \Delta t)
\end{array}\right]
$$

contains $M$ past channel estimations with a fixed time between the observations $\Delta t$. The filter coefficients

$$
\boldsymbol{\theta}=\boldsymbol{R}_{\mathrm{NLOS}}^{-1} \boldsymbol{r}_{\mathrm{NLOS}}
$$

are calculated from the cross-covariance between channel coefficient and observations $\boldsymbol{r}_{\mathrm{NLOS}}$ and the autocovariance matrix $\boldsymbol{R}_{\mathrm{NLOS}}$ of the observations according to

$$
\begin{gathered}
{\left[\boldsymbol{r}_{\mathrm{NLOS}}\right]_{j}=2 \sigma^{2} J_{0}\left(2 \pi f_{\mathrm{m}}\left(t_{\mathrm{p}}+(j-1) \Delta t\right)\right),} \\
{\left[\boldsymbol{R}_{\mathrm{NLOS}}\right]_{i j}=\left\{\begin{array}{ll}
2 \sigma^{2} J_{0}\left(2 \pi f_{\mathrm{m}}|j-i| \Delta t\right), & i \neq j \\
2 \sigma^{2}+2 \sigma_{n^{\prime}}^{2}, & i=j
\end{array} .\right.}
\end{gathered}
$$

To design the Wiener filter, knowledge about the variance $2 \sigma^{2}$, the maximum Doppler frequency $f_{\mathrm{m}}$ and the noise variance $2 \sigma_{\mathrm{n}}^{\prime 2}$ is needed. However, knowledge of these parameters is only required in a model-based analysis, which we concentrate on in this article, and not in measured fading channels, since the autocovariance is directly estimated in this case anyway. Therefore, we are saving efforts and do not introduce estimators for these parameters. Instead, we assume that they are known throughout the article. Using the outage predictor for measured fading channels is beyond the scope of this article and instead left for future work.

\subsubsection{Comparison with threshold}

Since we are interested if an up-state or an outage will occur, the predicted I/Q channel coefficient is compared with the threshold $\left|h_{\min }^{\prime}\right|$. Our idea is to choose a different threshold value for the predicted channel coefficient $\left|h_{\min }^{\prime}\right|$ and not the threshold in the two state fading model $\left|h_{\min }\right|$. This idea is depicted in Fig. 5 .

By using the threshold $\left|h_{\text {min }}^{\prime}\right|$ for the prediction, we are able to adjust the trade-off between the effective outage probability and the probability for an up-state prediction as discussed in Sect. 5. The objective is to get a more conservative predictor, such that falsely predicted up-states are rare, which allows the predictor to be used for URLLC scheduling. In return, falsely predicted outages occur more frequently, which the scheduler has to deal with. Numerical evaluation of this trade-off is presented in Sect. 7.3.

\subsubsection{Prediction error analysis}

For the given assumptions in Sect. 4 it can be shown that the prediction error

$$
e(t)=h\left(t+t_{\mathrm{p}}\right)-\hat{h}\left(t+t_{\mathrm{p}} \mid t\right)
$$

follows a zero mean complex Gaussian distribution 


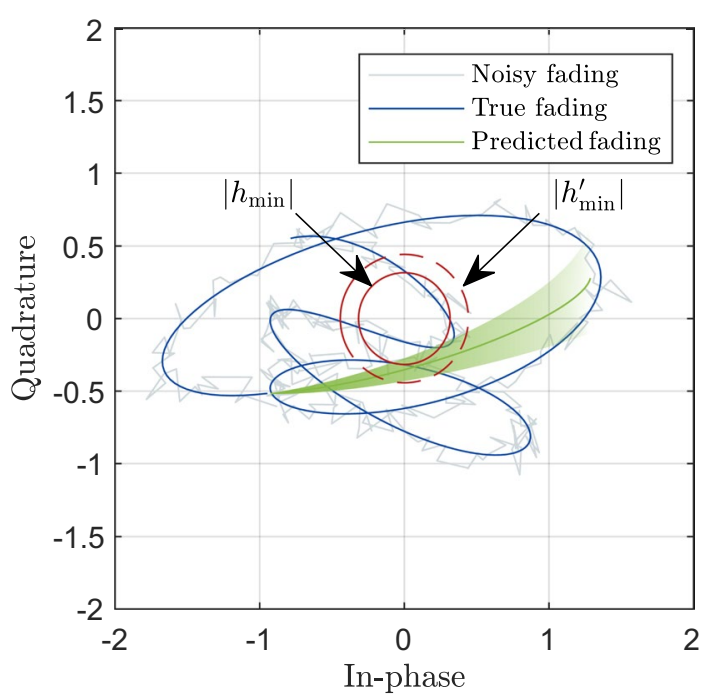

Fig. 5 Outage prediction concept. A threshold for the prediction $\left|h_{\min }^{\prime}\right|$ different from the outage threshold $\left|h_{\text {min }}\right|$ is introduced to tune the prediction uncertainty

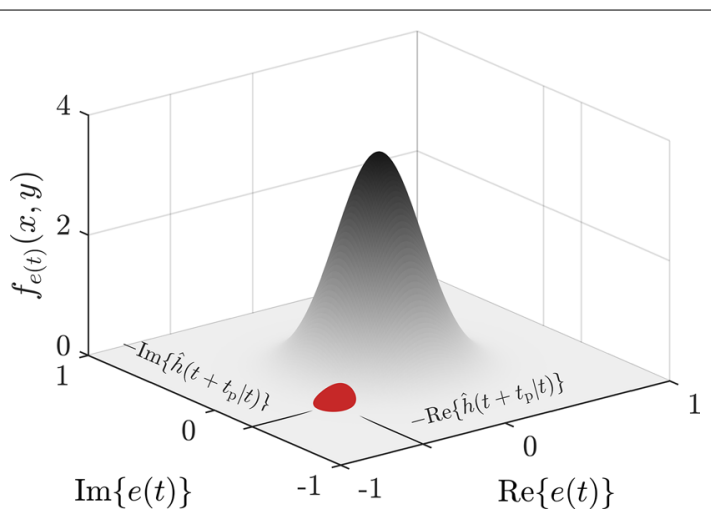

Fig. 6 Illustration of the integration area to calculate the future outage probability. The red circle marks the integration area and has radius $\left|h_{\min }\right|$

$$
e(t) \sim \mathcal{C N}\left(0,2 \sigma_{\mathrm{e}}^{2}\right)
$$

This originates from the fact that both $h(t)$ and $\hat{h}\left(t+t_{\mathrm{p}} \mid t\right)$ are zero mean complex Gaussian distributed and therefore also their difference follows a zero mean complex Gaussian distribution. Similarly, the filtering operation in (16) does not change the distribution type, as scaled and summed zero mean complex Gaussian random variables are again zero mean complex Gaussian. Thus, the distribution of (22) is completely parameterized by the variance of the prediction error [12]

$$
2 \sigma_{\mathrm{e}}^{2}=\mathrm{IE}\left[|e(t)|^{2}\right]=1-\boldsymbol{r}_{\mathrm{NLOS}}^{\mathrm{T}} \boldsymbol{R}_{\mathrm{NLOS}}^{-1} \boldsymbol{r}_{\mathrm{NLOS}}
$$

Knowing the distribution of the prediction error, a predicted channel coefficient value can now be associated with the probability of outage. We denote the 
probability for a future outage given a certain predicted channel coefficient $\hat{h}\left(t+t_{\mathrm{p}} \mid t\right)$ as $\operatorname{Pr}$ (future outage). As illustrated in Fig. 6, a future outage occurs when the prediction error $e(t)$ lies in the complex plane within an area $S$ of a circle around $-\hat{h}\left(t+t_{\mathrm{p}} \mid t\right)$ with radius $\left|h_{\min }\right|$. This is because the sum of predicted channel coefficient $\hat{h}\left(t+t_{\mathrm{p}} \mid t\right)$ and prediction error $e(t)$ is the true value of the future fading (rearranged version of (21)). Consequently, for a prediction error within the area $S$ the true channel coefficient lies within the outage region. Therefore, $\operatorname{Pr}$ (future outage) is determined by an area integral over the area $S$ according to

$$
\operatorname{Pr}(\text { future outage })=\int_{S} f_{e(t)}(x, y) \mathrm{d} S .
$$

Here, $f_{e(t)}(x, y)$ is the zero mean bivariate Gaussian probability density with variance $\sigma_{\mathrm{e}}^{2}$ for both dimensions I and Q. As there is no closed-form solution available for this integral, it must be evaluated numerically.

\subsection{Rician fading prediction}

We now extend the outage predictor for the more general Rician fading, where not only NLOS fading, but also a LOS component is present. The structure of the outage predictor for the Rician fading case is presented in Fig. 7.

Rician fading has a nonzero I/Q mean generated by the LOS-component, which is incompatible with a Wiener filter prediction. Therefore, the strategy when dealing with nonzero mean processes is to subtract the mean before filtering and adding the mean back again at the Wiener filter output [27]. As the time varying LOS-component can hardly be assumed to be known, the outage predictor in the Rician fading case has to employ estimators for the LOS parameters $A, f_{\mathrm{D}, \mathrm{LOS}}$ and $\varphi_{0}$ as a first step. The estimated LOS parameters lead to the full description of the LOS component at time $t$. After subtracting it from the history of channel estimations, the filter coefficients are calculated

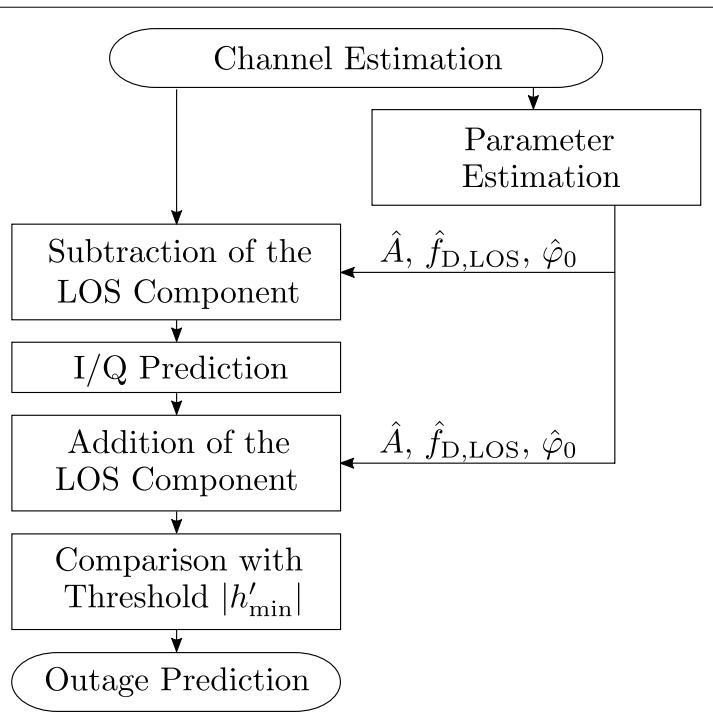

Fig. 7 Structure of the outage predictor for Rician fading 
and the NLOS component can be predicted equal to the Rayleigh fading case. In parallel, the LOS parameters are used to calculate a prediction of the LOS component at time $t+t_{\mathrm{p}}$ which can then be added to the Wiener filter output. This leads to a predicted I/Q channel sample, which can be thresholded against $\left|h_{\min }^{\prime}\right|$ to obtain an outage prediction. All steps from the LOS parameter estimation to the comparison with the threshold, are repeated when a new prediction needs to be calculated.

When comparing the outage predictor in Fig. 7 with Fig. 4, it can be seen that for Rician fading no outage probability is calculated. This is due to the fact, that it is not possible to analytically calculate the error distribution of the introduced LOS parameter estimation. The result is that also the distribution of the prediction error is unknown and outage probabilities cannot be calculated. The same problem arises when measured fading is predicted and the assumptions about the distributions do not hold anymore. In the rest of this section, we explain the individual predictor elements shown in Fig. 7 in detail.

\subsubsection{Parameter estimation}

Different from the Rayleigh fading case, a time varying LOS component is present and the parameters $A, f_{\mathrm{D}, \mathrm{NLOS}}, \varphi_{0}$ need to be estimated from a history of channel estimations.

When considering (10) as an estimation problem, where $2 \sigma^{2}, A$ and $f_{\mathrm{D}, \mathrm{LOS}}$ are the unknown parameters, both the CWGN $n^{\prime}(t)$ and the random NLOS component $\sqrt{2} \sigma \cdot h_{\mathrm{NLOS}}(t)$ act as noise. We neglect the temporal correlation of the NLOS component $\sqrt{2} \sigma \cdot h_{\mathrm{NLOS}}(t)$ for parameter estimation, since the complexity of the derivation is lower and the resulting estimators still perform very well in our outage prediction usecase. With both the CWGN and the NLOS component being complex Gaussian distributed the sum is complex Gaussian, too, and can be combined into a single variable.

This leads to the standard problem of estimating the parameters of a complex sinusoid in CWGN, which can be tackled using a ML estimation approach as shown in [25]. For the special case of noiseless Rician fading the desired ML estimators were derived in [28]. In the following, we adapt the estimators from [28] and employ an optimization to the frequency estimation.

A ML estimation of the frequency

$$
\hat{f}_{\mathrm{D}, \mathrm{LOS}}=-\arg \max \left(\frac{\left|\boldsymbol{\varphi}^{\prime} \boldsymbol{e}^{T}\right|^{2}}{\boldsymbol{e} \boldsymbol{e}^{H}}\right)
$$

is found by maximizing the periodogram with respect to $f_{\mathrm{D}, \mathrm{LOS}}$. Since the periodogram is the square of a discrete Fourier transform (DFT), a practical implementation would utilize the fast Fourier transform (FFT) algorithm. The observation vector of the LOS estimation

$$
\varphi^{\prime}=\left[\begin{array}{llll}
\hat{h}(t-(N-1) \Delta t) & \ldots & \hat{h}(t-\Delta t) & \hat{h}(t)
\end{array}\right]
$$

consists of $N$ values and is sampled at a discrete sampling period $\Delta t$. Furthermore, a vector of exponential terms 


$$
\boldsymbol{e}=\left[\begin{array}{llll}
\exp \left(-\mathrm{j}\left(2 \pi \mathrm{f}_{\mathrm{D}, \mathrm{LOS}}(\mathrm{N}-1) \Delta \mathrm{t}\right)\right) & \ldots & \exp \left(-\mathrm{j}\left(2 \pi \mathrm{f}_{\mathrm{D}, \mathrm{LOS}} \Delta \mathrm{t}\right)\right) & 1
\end{array}\right]
$$

is part of the estimator. Since the true value of the LOS Doppler frequency $f_{\mathrm{D}, \mathrm{LOS}}$ could be located between the bins of the periodogram, the frequency estimation can be greatly improved by interpolation as shown in [29]. The authors propose an iterative approach, which we also employ in this article to refine the frequency estimate (25). As suggested by the authors, we also use two iterations.

The ML estimates for the remaining parameters can then be calculated by inserting the frequency estimate in (27) (we denote this vector as $\hat{\boldsymbol{e}}$ in the following) and using

$$
\begin{aligned}
& \hat{A}=\left|\frac{\boldsymbol{\varphi}^{\prime} \hat{\boldsymbol{e}}^{H}}{\hat{\boldsymbol{e}} \hat{\boldsymbol{e}}^{H}}\right|, \\
& \hat{\varphi}_{0}=\arg \left\{\frac{\boldsymbol{\varphi}^{\prime} \hat{\boldsymbol{e}}^{H}}{\hat{\boldsymbol{e}} \hat{\boldsymbol{e}}^{H}}\right\} .
\end{aligned}
$$

The estimator (29) yields a phase estimate of the last element in (26), which is preferable from a prediction point of view. Combining the estimates $f_{\mathrm{D}, \mathrm{LOS}}, \hat{A}$ and $\hat{\varphi}_{0}$ gives an estimate of the LOS component

$$
\hat{A} \cdot \hat{h}_{\mathrm{LOS}}(t)=\hat{A} \cdot \exp \left(\mathrm{j}\left(2 \pi \hat{f}_{\mathrm{D}, \mathrm{LOS}}\left(t-t_{0}\right)+\hat{\varphi}_{0}\right)\right) .
$$

\subsubsection{I/Q prediction and comparison with threshold}

With the available parameter estimations, a prediction of the I/Q channel coefficients in the Rician fading case can be performed. Since we are again using a Wiener Filter which relies on the input to be zero mean, a prediction of future I/Q channel samples

$$
\hat{h}\left(t+t_{\mathrm{p}} \mid t\right)=\left(\boldsymbol{\varphi}-\hat{A} \cdot \hat{\boldsymbol{h}}_{\mathrm{LOS}}\right) \boldsymbol{\theta}+\hat{A} \cdot \hat{h}_{\mathrm{LOS}}\left(t+t_{\mathrm{p}}\right)
$$

consists of the estimated LOS component at prediction time $\hat{A} \cdot \hat{h}_{\mathrm{LOS}}\left(t+t_{p}\right)$ added to the FIR filter output $\left(\boldsymbol{\varphi}-\hat{A} \cdot \hat{\boldsymbol{h}}_{\mathrm{LOS}}\right) \boldsymbol{\theta}$, with the observation vector of the Wiener filter $\boldsymbol{\varphi}$ being adjusted for the estimate of the LOS component vector

$$
\hat{A} \cdot \hat{\boldsymbol{h}}_{\mathrm{LOS}}=\hat{A} \cdot\left[\begin{array}{llll}
\hat{h}_{\mathrm{LOS}}\left(t_{0}\right) & \hat{h}_{\mathrm{LOS}}\left(t_{0}-\Delta t\right) & \ldots & \hat{h}_{\mathrm{LOS}}\left(t_{0}-(M-1) \Delta t\right)
\end{array}\right]
$$

at the input of the filter. Since after subtraction of the LOS component only the NLOS fading remains, the filter coefficient can be calculated in the same way as in the Rayleigh fading case, shown in Sect. 6.1.1. Also, the comparison with the threshold is no different from the Rayleigh fading case in Sect. 6.1.2.

\section{Results and discussion}

In this section, the performance of the outage predictor for the Rayleigh and the Rician fading case is analyzed numerically. The scenario and the chosen parameters for numerical evaluation are described in Sect. 7.1. After investigating the influence 
of the predictor parameters in Sect. 7.2, the performance evaluation of the outage prediction schemes is conducted in Sect. 7.3.

\subsection{Scenarios}

The numerical evaluation is conducted for selected numerical values which are summarized in Table 1 . In the following, three different $K$-factors are investigated to reflect the case of a strong LOS component $(K=10)$, a medium LOS component $(K=5)$ and no LOS component ( $K=0$, Rayleigh fading case). The performance is shown for two different mean channel estimation SNRs of $20 \mathrm{~dB}$ and $10 \mathrm{~dB}$. In both cases, the fading margin is set to $F=10 \mathrm{~dB}$. All plots in the following sections are generalized by using times that are normalized to the maximum Doppler frequency $f_{\mathrm{m}}$. This allows the results to be used for evaluation of various applications without the need of re-simulation. To put the provided normalized plots into perspective, the example of a remote-controlled automated guided vehicle (AGV) in an industrial campus network is considered. For this use-case we assume a constant relative velocity $v=0.8 \mathrm{~m} / \mathrm{s}$ and a carrier frequency $f_{\mathrm{c}}=3.75 \mathrm{GHz}$. According to $f_{\mathrm{m}}=\frac{f_{\mathrm{c}} \cdot v}{c}$, where $c$ is the speed of light, this yields a maximum Doppler shift of $10 \mathrm{~Hz}$. In the Rician fading case, the LOS Doppler frequency $f_{\mathrm{D}, \mathrm{LOS}}$ and the starting phase of the LOS component $\varphi_{0}$ were varied randomly.

\subsection{Parameter analysis}

Before being able to investigate the performance of the outage prediction schemes, the predictor requires parameterization. The Wiener filter is parameterized by its history length $M$ and its sampling period $\Delta t$. Furthermore, the history length $N$ of the LOS estimator needs to be specified. In the following, an analysis of these parameters is conducted for the scenario described in Sect. 7.1 to obtain a satisfactory configuration for the following performance evaluation. Throughout the whole section, the normalized prediction horizon $t_{\mathrm{p}} f_{\mathrm{m}}$ is arbitrary set to 0.1 . The results of this section were generated by means of computer simulation according to the Monte-Carlo approach. For each point $4 \times 10^{5}$ predicted fading samples were compared with the respective ideal future fading value.

\subsubsection{Sampling period}

In Fig. 8, the mean squared error (MSE) of the I/Q prediction is plotted against the normalized sampling period $\Delta t f_{\mathrm{m}}$ for a fixed history length of the Wiener filter and the LOS estimator. Although the MSE is not suitable to describe the outage prediction performance directly, it can be used as a performance indicator. Generally

Table 1 Values for numerical evaluation

\begin{tabular}{ll}
\hline Parameter & Value \\
\hline Rician $K$ factor & $0,5,10$ \\
Mean channel estimation SNR & $20 \mathrm{~dB}, 10 \mathrm{~dB}$ \\
Fading margin $F$ & $10 \mathrm{~dB}$ \\
\hline
\end{tabular}




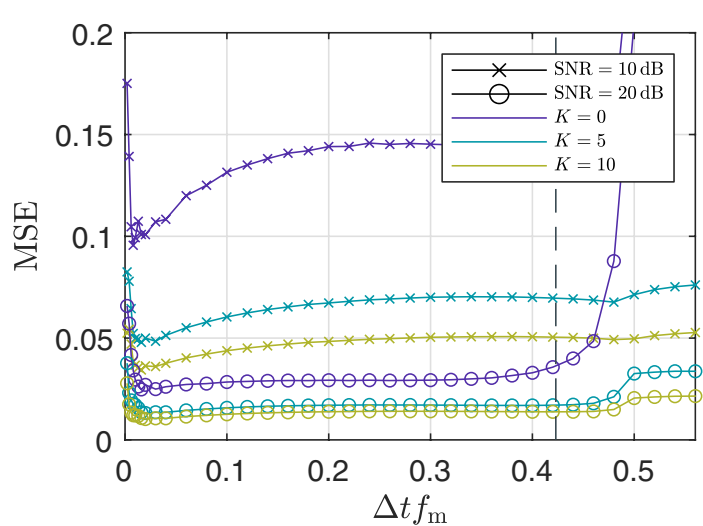

Fig. 8 Influence of the sampling period $\Delta t$ on the MSE of the prediction. $F=10 \mathrm{~dB}, M=25, N=128$ and $t_{\mathrm{p}} f_{\mathrm{m}}=0.1$; the dashed line is an empirical value for the coherence time [6]

speaking, the higher the error of the I/Q prediction, the worse the outage prediction performance after comparing the predicted I/Q channel coefficient with the threshold $\left|h_{\text {min }}^{\prime}\right|$. When looking at the curves in Fig. 8, it can be seen that very small as well as very large sampling periods do not perform well for the investigated SNRs and $K$ factors. For a fixed history length, very small sampling periods $\Delta t$ result in the observation not spanning enough to capture the continuous variation of the fading. Similarly, very large sampling periods $\Delta t$, which are greater than the coherence time, lead to uncorrelated observations. According to the popular rule of thumb from [6], the coherence time $t_{\text {coh }}$ can be approximated as $t_{\text {coh }}=\frac{0.423}{f_{\mathrm{m}}}$, which is close to the point in Fig. 8 where the MSE begins to rise steeply. In all cases, a long plateau of the MSE can be observed, where a wide range of sampling periods $\Delta t$ perform almost equally well. In case of a small SNR of $10 \mathrm{~dB}$ and especially for Rayleigh fading $(K=0)$, a clear optimum for the sampling period arises. For practical systems, a sampling period between this optimum and the coherence time could be chosen. The choice of the sampling period is a trade-off: For scheduling purposes, large sampling periods unavoidably lead to large prediction horizons, which generally lead to worse prediction

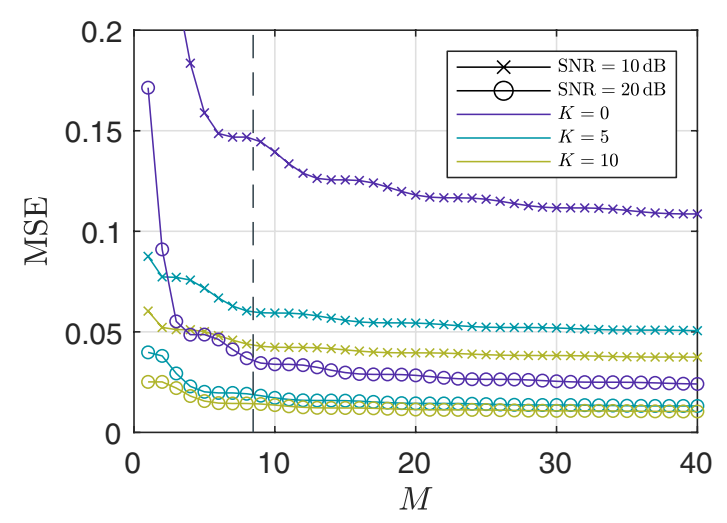

Fig. 9 Influence of the Wiener filter history length $M$ on the MSE of the prediction. $F=10 \mathrm{~dB}, \Delta t f_{\mathrm{m}}=0.05$ and $N=128$; the dashed line is the point where $M \Delta t$ equals an empirical value for the coherence time [6] 
performance than short-term predictions. On the other hand, small sampling periods imply that training signals need to be sent more frequently. This, however, has a negative impact on the efficiency of the communications system and the number of users which can be allocated to send these training signals. For numerical evaluation, we settled on a normalized sampling period of $\Delta t f_{\mathrm{m}}=0.05$. This equals a sampling period of $\Delta t=5 \mathrm{~ms}$ in the AGV use case described in Sect. 7.1.

\subsubsection{Wiener filter history length}

In Fig. 9, the Wiener filter history length $M$ is investigated. Throughout all curves, an increase in the history length $M$ results in a reduction in the MSE. This is intuitive as adding more information to the estimation will not lead to degradation. However, the performance gain lowers with increasing $M$, which is also intuitive as recent samples carry more information about the future channel state compared to outdated samples. However, in the case of a very low $K$ factor of $K=0$, high values of $M$ still improve the performance. For higher $K$ factors, as the LOS component dominates the NLOS component, the curves become more flat even for small Wiener filter history lengths $M$. Since the Wiener filter is responsible for the NLOS prediction, the choice of $M$ becomes less relevant for these high $K$ factors. A good rule of thumb is to choose $M$ such that $M \Delta t$ is larger than the coherence time. Throughout our numerical evaluation, we settle for a Wiener filter history length of $M=25$.

\subsubsection{LOS estimation history length}

Finally, in Fig. 10 the MSE is plotted for different history lengths of the LOS estimator $N$. Similar to the history length of the Wiener filter $M$, high values of $N$ are beneficial for the performance of the predictor. Again, the steepness of the curves decreases with rising $N$, thus, after a certain value the increase in $N$ does not lead to a significant performance increase anymore. Due to complexity minimization, $N$ should be kept as low as possible as the calculation of the FFT is required in (25). For numerical evaluation of the Rician fading case, we settled for $N=128$.

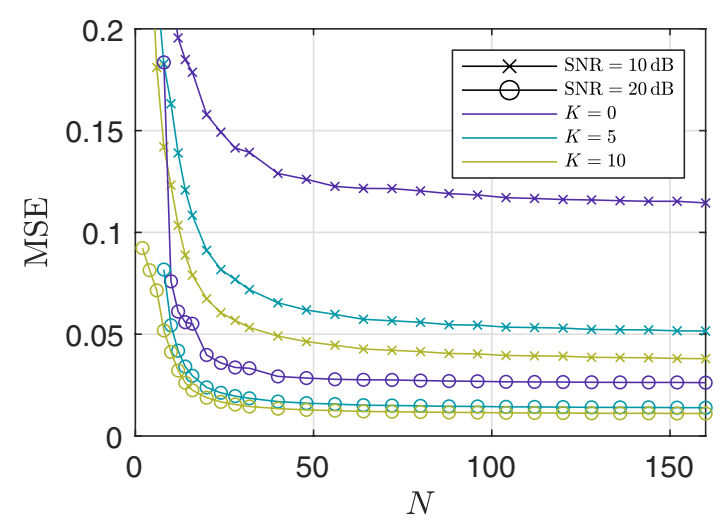

Fig. 10 Influence of the LOS estimation history length $N$ on the MSE of the prediction. $F=10 \mathrm{~dB}$, $\Delta t f_{m}=0.05$ and $M=25$ 


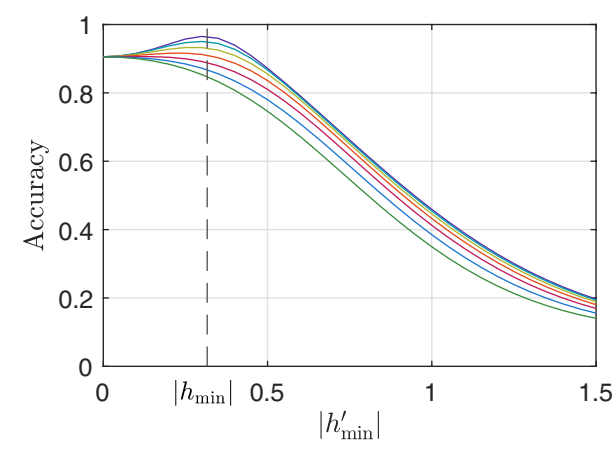

a Accuracy

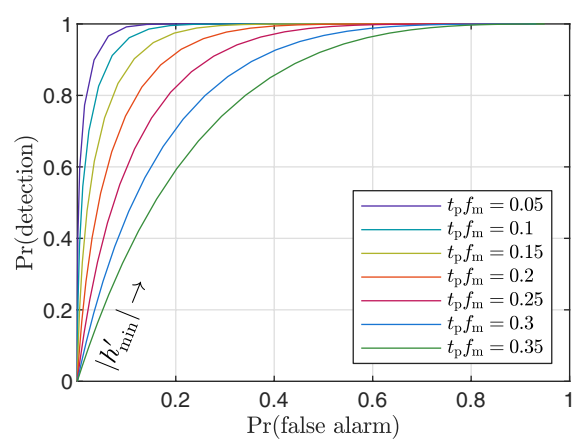

b Receiver operating characteristic curves

Fig. 11 Analysis of the outage prediction performance using common binary classification metrics. $20 \mathrm{~dB}$ $\mathrm{SNR}, F=10 \mathrm{~dB}, \Delta t f_{\mathrm{m}}=0.05$ and $M=25$

\subsection{Numerical evaluation}

With the scenario from Sect. 7.1 and the predictor parameters from Sect. 7.2, the prediction schemes can be evaluated numerically. In the following, we conduct the evaluation for the Rayleigh fading outage predictor and the Rician fading outage predictor separately as they have different feature sets.

\subsubsection{Rayleigh fading prediction}

We first investigate the performance of the outage prediction, which is one of the two predictor outputs. The shown values originate from Monte-Carlo simulations. In our simulations, $2 \cdot 10^{8} \mathrm{I} / \mathrm{Q}$ channel coefficients were fed into the outage predictor for each prediction horizon and compared with the true future fading state. To put this into perspective, at a sampling period of $5 \mathrm{~ms}$, which is used in the AGV scenario, this equals 11.6 days of consecutive fading. Two examples for classical metrics to investigate our binary classification problem were introduced in Sect. 5 and are plotted in Fig. 11 for different prediction horizons. In Fig. 11a the accuracy of the outage predictor is plotted against different threshold values for the prediction $h_{\min }^{\prime}$. One can see that an optimal accuracy arises for small prediction horizons $t_{\mathrm{p}}$ when the threshold for prediction $h_{\min }^{\prime}$ is chosen near the actual outage threshold $h_{\min }$. This might appear appealing for the choice of $h_{\mathrm{min}}^{\prime}$, however, the optimum and the metric as a whole have only little practical relevance for the outage predictor. The metric combines both error types (false positives and false negatives) within a single number. However, false positives are considerably more critical for an URLLC scheduler as they are the defining parameter for the QoS. Even worse, as we tune the outage predictor to be more conservative by increasing $h_{\min }^{\prime}$, the number of false positives is orders of magnitude smaller than the number of false negatives. Therefore, the accuracy of the predictor almost only reflects false negatives, which is the dominating error type in this case. A more informative performance evaluation of the predictor can be done by studying the receiver operating characteristic (ROC) curves, which are shown in Fig. 11b. In this performance figure the probability of detection $\operatorname{Pr}$ (detection) is plotted against the probability for a false alarm $\operatorname{Pr}$ (false alarm). The threshold value for the prediction $\left|h_{\text {min }}^{\prime}\right|$ now shifts the operating point in the ROC. We can learn from the ROC curves that, when increasing $\left|h_{\min }^{\prime}\right|$ we get a more conservative 


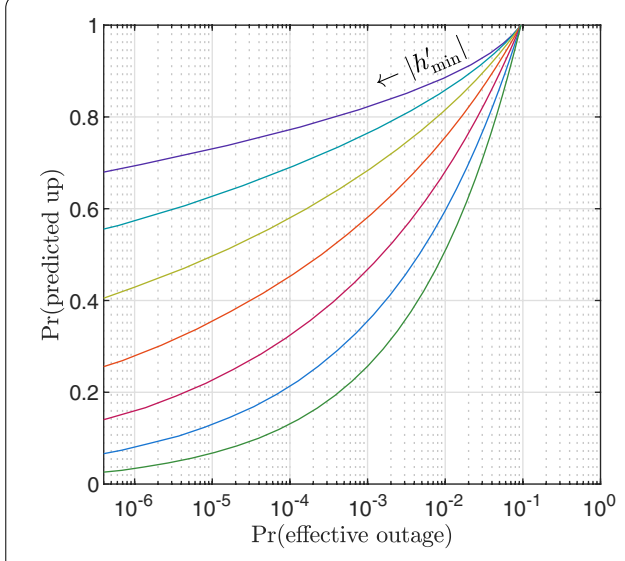

a $20 \mathrm{~dB} \mathrm{SNR}$

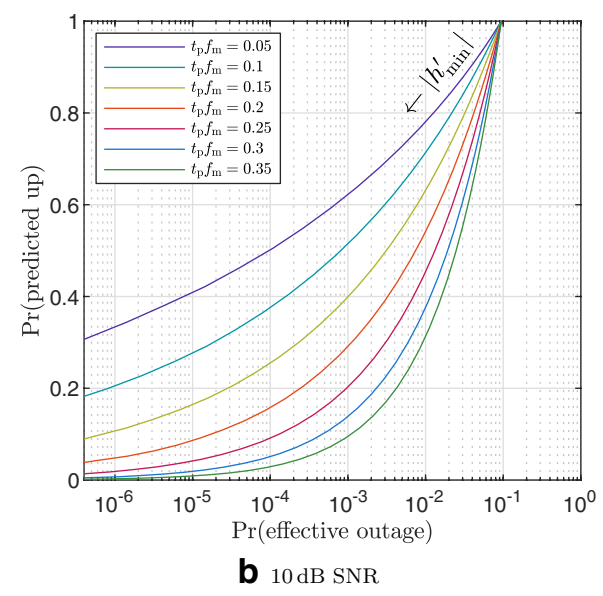

b $10 \mathrm{~dB}$ SNR

Fig. 12 Analysis of the outage prediction performance. $F=10 \mathrm{~dB}, \Delta t f_{m}=0.05$ and $M=25$

predictor so that outages are more likely to be detected at the cost of more false alarms. However, we are still not able to make qualitative statements about the expected scheduling performance since we are not able to evaluate which combination of $\operatorname{Pr}$ (detection) and $\operatorname{Pr}$ (false alarm) is acceptable in the context of URLLC radio resource scheduling.

Therefore, following our discussion in Sect. 5, we use $\operatorname{Pr}$ (effective outage) instead of $\operatorname{Pr}$ (false alarm). This metric shows the effective outage probability of a perfect scheduler and thus can be used to qualitatively evaluate the risk of fatal failures due to prediction errors. Instead of $\operatorname{Pr}$ (detection) we use $\operatorname{Pr}$ (predicted up) to evaluate the percentage of time the observed link can be utilized for URLLC traffic of a specific UE. The performance curves with these metrics are plotted in Fig. 12. Similar to the ROC curves, each line spans different operating points, which can be adjusted by varying the threshold $\left|h_{\min }^{\prime}\right|$. A prominent point in these curves is $\left|h_{\min }^{\prime}\right|=0$, where the channel is predicted as up $100 \%$ of the time and the effective outage probability equals the average outage probability. The average outage probability for Rayleigh fading can be calculated using $\operatorname{Pr}($ outage $)=1-\exp (-1 / F)$ [30]. In Fig. 12a the results for a mean channel estimation SNR of $20 \mathrm{~dB}$ are shown and discussed using the AGV scenario with $f_{\mathrm{m}}=10 \mathrm{~Hz}$. If, for instance, a prediction horizon of $t_{\mathrm{p}}=5 \mathrm{~ms}$ is needed to overcome the delay $\tau$ between monitoring and payload and an effective outage probability $\operatorname{Pr}$ (effective outage) $=10^{-3}$ is targeted, the link can be used approximately $82 \%$ of the time for URLLC traffic. If a higher prediction horizon of $t_{\mathrm{p}}=10 \mathrm{~ms}$ is required and the same effective outage probability of $\operatorname{Pr}$ (effective outage) $=10^{-3}$ is targeted, the link can only be utilized approximately $76 \%$ of the time. In Fig. 12b, a lower mean channel estimation SNR of $10 \mathrm{~dB}$ is shown. The lower SNR leads to a worse overall performance, e.g., when using our previous example with $t_{\mathrm{p}}=5 \mathrm{~ms}$ and $\operatorname{Pr}$ (effective outage $)=10^{-3}$, the probability of having a predicted up link is only $62 \%$ instead of $82 \%$. An increase in the maximum Doppler frequency, resulting for example from a higher carrier frequency $f_{\mathrm{c}}$ or an increasing movement speed $v$, will reduce the achievable prediction horizon $t_{\mathrm{p}}$ for a set of $\operatorname{Pr}$ (effective outage) and $\operatorname{Pr}$ (predicted up). As a result it can be concluded, that the proposed prediction approach is of limited benefit for realizing URLLC services in future mmWave and terahertz communications systems. 


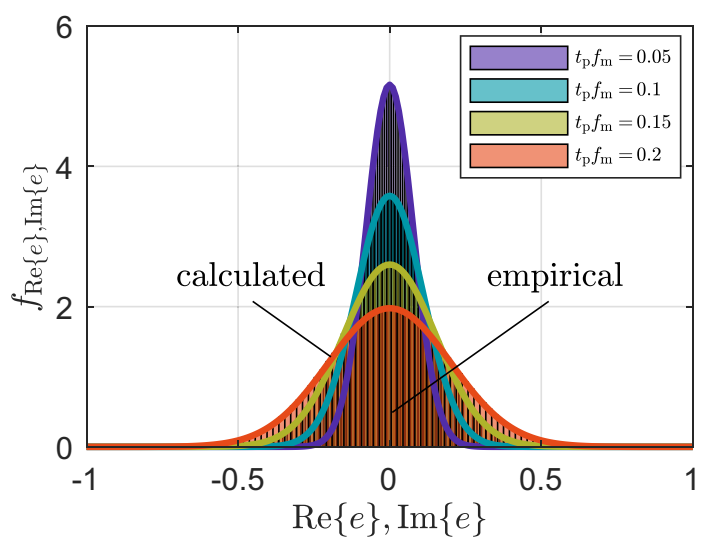

Fig. 13 Validation of the prediction error distribution

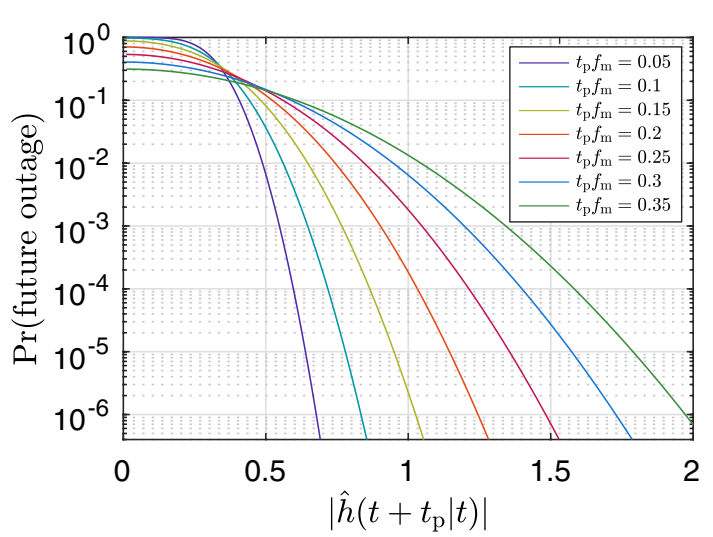

Fig. 14 Future outage probability depending from the prediction amplitude. $20 \mathrm{~dB} S N R, F=10 \mathrm{~dB}$, $\Delta t f_{m}=0.05$ and $M=25$

Additional to the prediction of outages, the Rayleigh fading outage predictor is able to calculate future outage probabilities under the assumptions discussed in Sect. 6.1. Basis for the calculation is (22), which states that the real and imaginary parts of the prediction error follow a zero mean Gaussian distribution. The variance of the zero mean Gaussian distribution was calculated in (23). These findings are validated in Fig. 13, where an empirical estimate (a normalized histogram) of the probability density is compared with the analytical calculation for different prediction horizons. One can see that the empirical histograms fit very well beneath the calculated probability densities.

With the known error distribution, the probability for a future outage can be calculated over the area integral (24). An analysis of this integral reveals that the probability for a future outage only depends on the amplitude of the predicted fading $\left|\hat{h}\left(t+t_{\mathrm{p}} \mid t\right)\right|$. The resulting future outage probabilities are plotted in Fig. 14. Using the AGV scenario with $f_{\mathrm{m}}=10 \mathrm{~Hz}$, if a channel coefficient with an absolute value of 0.6 is predicted at a prediction horizon of $t_{\mathrm{p}}=5 \mathrm{~ms}$, the channel state would be in the outage region with a probability of $10^{-4}$. If, instead, the distance of the predicted value from the origin is 0.7 , the probability is $2 \times 10^{-7}$ and therefore approximately three orders of magnitude 

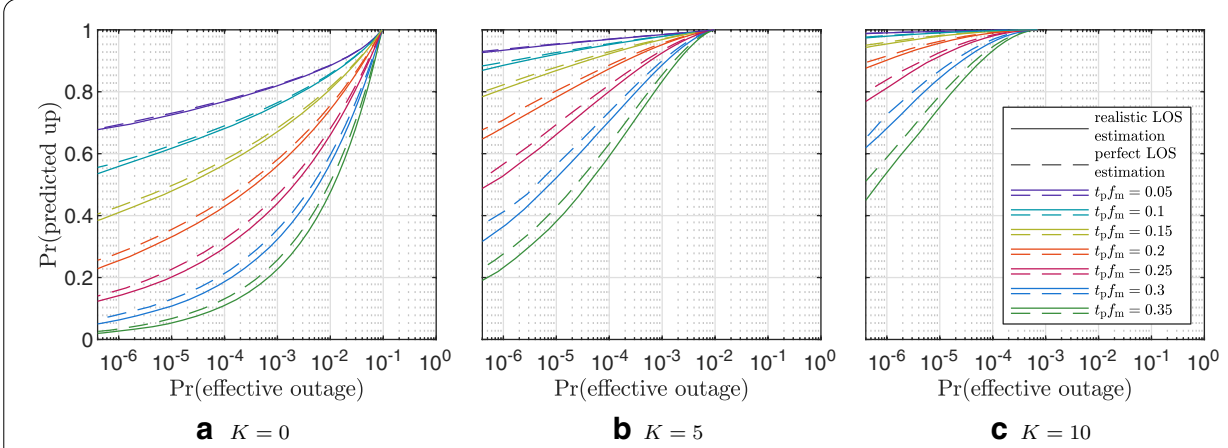

Fig. 15 Analysis of the outage prediction performance. $20 \mathrm{~dB} S N R, F=10 \mathrm{~dB}, \Delta t f_{m}=0.05, M=25$ and $N=128$

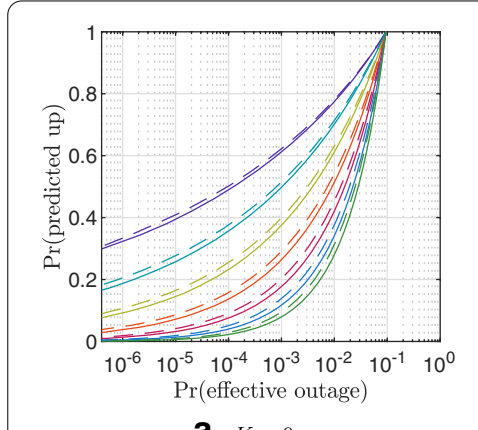

a $K=0$

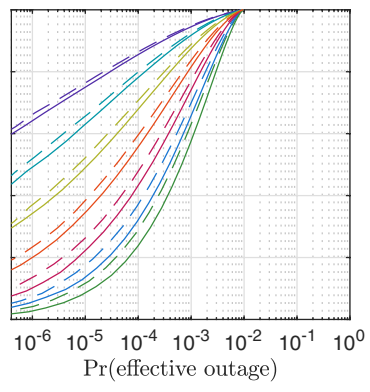

b $K=5$

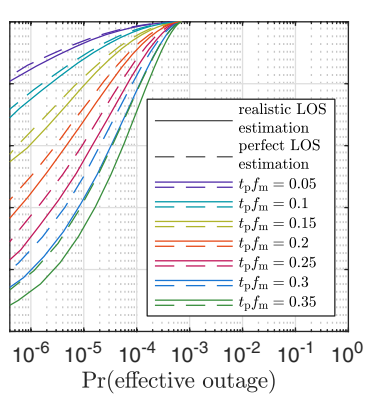

C $K=10$

Fig. 16 Analysis of the outage prediction performance. $10 \mathrm{dBSNR}, F=10 \mathrm{~dB}, \Delta t f_{\mathrm{m}}=0.05, M=25$ and $N=128$

smaller. For higher prediction horizons $t_{\mathrm{p}}$, the predicted channel coefficient has to be farther away from the origin to achieve the same outage probabilities. This is due to the fact that the variance of the error distribution is higher.

\subsubsection{Rician fading prediction}

The performance curves of the outage predictor described in Sect. 6.2 for the more general Rician fading case are shown as solid lines in Fig. 15 for a SNR of $20 \mathrm{~dB}$ and in Fig. 16 for a SNR of $10 \mathrm{~dB}$. Each line is based on $2 \times 10^{8}$ predicted I/Q channel coefficients. To investigate the influence of the LOS estimation, which is the novel component compared to the Rayleigh fading case, we also show the case of ideal LOS parameter estimation as dashed lines, where ideal estimates are used for subtraction and prediction of the LOS component and only the NLOS fading is realistically predicted using the Wiener filter. Therefore, the dashed lines for $K=0$ correspond to the performance curves discussed in Fig. 12. The performance loss which can be observed between solid and dashed lines originates from the imperfections of the introduced LOS estimator. Overall, the performance loss is the smallest when $t_{\mathrm{p}}$ is small and the SNR is high. For large prediction horizons and a small SNR the performance loss is more pronounced; however, the curves are still close to their ideal counterparts. 
When comparing the same prediction horizons for different $K$ factors, one can observe that the outage predictor performs better at high $K$ factors. For example, when in the AGV scenario a prediction horizon $t_{\mathrm{p}}=10 \mathrm{~ms}$ is utilized in case of $K=0$ and the effective outage target probability is set to $\operatorname{Pr}$ (effective outage) $=10^{-5}$, the observed link is only predicted as up with a $62 \%$ probability. However, for $K=5$ the same prediction horizon and effective probability is achieved while the channel is predicted as up with a much higher probability of $92 \%$ and for $K=10$ even $99 \%$ is reached. A reason for that is the decrease in randomness in the fading for increasing $K$ factors. The randomness originates from the NLOS component, whose impact is reduced when a strong LOS component is present. Ultimately, the deterministic LOS component is easier to predict resulting in a better outage prediction performance.

In Fig. 16, an overall worse performance can be observed compared to Fig. 15 due to the lower SNR. While high $K$ factors of $K=\{5,10\}$ still show a promising performance, for $K=0$ a small prediction horizon of $5 \mathrm{~ms}$ achieves $\operatorname{Pr}$ (effective outage) $=10^{-5}$ only with a predicted up probability of $39 \%$. To alleviate this behavior to some extent, the number of pilot symbols $P$ can be increased, although this leads to a reduced spectral efficiency. However, analyzing this trade-off is out of scope of this article and will be left for future work.

\section{Conclusion}

In view of reducing radio resource consumption for ultra-reliable wireless communication links, monitoring the fast fading channel and taking measures based on the predicted fading state is a promising strategy. This article provided outage prediction schemes for Rayleigh and Rician fading and introduced suitable metrics that describe their performance.

For the Rayleigh fading case and especially for small prediction horizons, the presented predictor features a low missed outage probability while simultaneously not rigorously denying the current channel. For the Rician fading case, i.e., with a LOS component present, a LOS estimator is utilized. Compared to the case where the LOS component is known, the LOS estimator only degrades prediction performance minorly. Generally in the presence of a LOS component, the outage prediction performance is improved to the Rayleigh fading case. Evidently, the LOS estimation comes at the cost of increased complexity, mainly due to the calculation of the FFT as part of the LOS Doppler estimation.

The results showed that under realistic conditions, gaining multiple orders of magnitude in transmission reliability is well within the scope of this channel prediction concept.

Further studies need to investigate the outage prediction performance under real world conditions to identify possible issues that are not covered by the Rayleigh and Rician fading models. To handle general fading assumptions in changing environments empirical covariance matrices and adaptive filtering algorithms to efficiently update the filter coefficients may be used. Ultimately, a scheduler needs to be developed that incorporates the channel prediction outcomes for every UE to an effective and highly reliable channel allocation. 


\begin{abstract}
Abbreviations
3GPP: 3rd Generation Partnership Project; 4G: Fourth generation; 5G: Fifth generation; 6G: Sixth generation; AGV: Automated guided vehicle; AR: Auto-regressive; BER: Bit error rate; BS: Base station; CWGN: Complex white Gaussian noise; DFT: Discrete Fourier transform; DL: Downlink; FFT: Fast Fourier transform; FIR: Finite impulse response; I/Q: In-phase and quadrature; LMMSE: Linear minimum mean square error; LMS: Least mean squares; LOS: Line-of-sight; LS: Least squares; ML: Maximum likelihood; MMSE: Minimum mean square error; MSE: Mean squared error; MVU: Minimum variance unbiased; NLMS: Normalized least mean squares; NLOS: Non-line-of-sight; OFDM: Orthogonal frequency-division multiplexing; QoS: Quality of service; RLS: Recursive least squares; ROC: Receiver operating characteristic; SISO: Single input single output; SNR: Signal/noise ratio; SOS: Sum-of-sinusoids; UE: User equipment; UL: Uplink; URLLC: Ultra-reliable low-latency communications.
\end{abstract}

\title{
Acknowledgements
}

We thank the Center for Information Services and High Performance Computing (ZIH) at Technische Universitat Dresden for generous allocations of computer time.

\section{Authors' contributions}

AT performed the simulations, generated the plots, and drafted the manuscript with input from all authors. ES drafted the section about literature research. GF and NF proposed to investigate the prediction of small-scale fading for URLLC and supervised the findings of this work. All authors contributed to the development of the idea and the interpretation of the results. All authors read and approved the final manuscript.

\section{Funding}

Open Access funding enabled and organized by Projekt DEAL. This work was in part funded by the German Research Foundation (DFG, Deutsche Forschungsgemeinschaft) as part of Germany's Excellence Strategy - EXC 2050/1 - Project ID 390696704 - Cluster of Excellence "Centre for Tactile Internet with Human-in-the-Loop" (CeTI) of Technische Universität Dresden. This work was also in part sponsored by the Federal Ministry of Education and Research (BMBF) within the program "Twenty20 - Partnership for Innovation" under contract 03ZZ0528E - "fast robotics", within the project VERITAS under the Project Number 01IS18073 and as part of the project "TACNET 4.0" under Grant 16KIS0719. This research was co-financed by public funding of the state of Saxony/Germany.

\section{Availability of data and materials}

Not applicable.

\section{Declarations}

\section{Competing interests}

The authors declare that they have no competing interests.

\section{Author details}

${ }^{1}$ Vodafone Chair Mobile Communications Systems, Technische Universität Dresden, Dresden, Germany. ${ }^{2}$ Centre for Tactile Internet with Human-in-the-Loop (CeTI), Dresden, Germany. ${ }^{3}$ Barkhausen Institut, Dresden, Germany.

Received: 2 November 2020 Accepted: 24 March 2021

Published online: 13 April 2021

\section{References}

1. N. Mahmood, O. López, O. Park, I. Moerman, K. Mikhaylov, E. Mercier, A. Munari, F. Clazzer, S. Böcker, H. Bartz, White paper on critical and massive machine type communication towards $6 \mathrm{G}$

2. W. Saad, M. Bennis, M. Chen, A vision of $6 \mathrm{G}$ wireless systems: applications, trends, technologies, and open research problems. IEEE Netw. 34, 134-142 (2020)

3. H. Lasi, P. Fettke, H. Kemper, T. Feld, M. Hoffmann, Industry 4.0. Bus. Inf. Syst. Eng. 6, 239-242 (2014)

4. G. Fettweis, The tactile internet: applications and challenges. IEEE Veh. Technol. Mag. 9, 64-70 (2014)

5. F. Fitzek, S. Li, S. Speidel, T. Strufe, M. Simsek, Tactile Internet with Human-in-the-Loop (Academic Press, Cambridge, 2021).

6. T. Rappaport, Wireless Communications: Principles and Practice (Prentice Hall, Upper Saddle River, 2002)

7. A. TraßI, L. Scheuvens, T. Hößler, E. Schmitt, N. Franchi, G. Fettweis, Outage prediction for URLLC in Rayleigh fading. In: Proceedings of the 29th European Conference on Networks and Communications: 16-17 June; Virtual, pp. 116-121 (2020)

8. A. TraßI, T. Hößler, L. Scheuvens, N. Schwarzenberg, G. Fettweis, Outage prediction for URLLC in Rician fading. In: Proceedings of the IEEE International Symposium on Personal, Indoor and Mobile Radio Communications: 13-16 September; Helsinki (2021). Submitted for publication

9. A. Duel-Hallen, $\mathrm{S}$. Hu, H. Hallen, Long range prediction of fading signals: enabling adaptive transmission for mobile radio channels. IEEE Signal Process. Mag. 17, 62-75 (2000)

10. J. Andersen, J. Jensen, S. Jensen, F. Frederiksen, Prediction of future fading based on past measurements. In: Proceedings of the 50th IEEE Vehicular Technologies Conference: 19-22 September; Amsterdam, pp. 151-155 (1999)

11. L. Dong, G. Xu, H. Ling, Prediction of fast fading mobile radio channels in wideband communication systems. In: Proceedings of the IEEE Global Telecommunications Conference: 25-29 November; San Antonio, pp. 3287-3291 (2001)

12. T. Ekman, Prediction of mobile radio channels - modeling and design. PhD thesis, Uppsala University, Department of Electrical Engineering (2002) 
13. T. Ekman, M. Sternad, A. Ahlen, Unbiased power prediction of Rayleigh fading channels. In: Proceedings of the 56th IEEE Vehicular Technology Conference: 24-28 September; Vancouver, pp. 280-284 (2002)

14. A. Duel-Hallen, H. Hallen, T. Yang, Long range prediction and reduced feedback for mobile radio adaptive OFDM systems. IEEE Trans. Wirel. Commun. 5, 2723-2733 (2006)

15. A. Duel-Hallen, Fading channel prediction for mobile radio adaptive transmission systems. Proc. IEEE $\mathbf{9 5}, \mathbf{2 2 9 9 - 2 3 1 3}$ (2007)

16. D. Schafhuber, G. Matz, MMSE and adaptive prediction of time-varying channels for OFDM systems. IEEE Trans. Wirel. Commun. 4, 593-602 (2005)

17. D. Kapoor, A. Kohli, Channel estimation and long-range prediction of fast fading channels for adaptive OFDM system. Int. J. Electron. 105, 1451-1466 (2018)

18. A. Heidari, A. Khandani, D. Mcavoy, Adaptive modelling and long-range prediction of mobile fading channels. IET Commun. 4, 39-50 (2010)

19. R. Liao, H. Wen, J. Wu, H. Song, F. Pan, L. Dong, The Rayleigh fading channel prediction via deep learning. Wireless Communications and Mobile Computations (2018)

20. Y. Zhu, X. Dong, T. Lu, An adaptive and parameter-free recurrent neural structure for wireless channel prediction. IEEE Trans. Commun. 67, 8086-8096 (2019)

21. V. Swamy, P. Rigge, G. Ranade, B. Nikolic, A. Sahai, Predicting wireless channels for ultra-reliable low-latency communications. In: Proceedings of the IEEE International Symposium on Information Theory: 17-22 June; Colorado, pp. 2609-2613 (2018)

22. V. Swamy, P. Rigge, G. Ranade, B. Nikolic, A. Sahai, Wireless channel dynamics and robustness for ultra-reliable lowlatency communications. Sel. Areas Commun. 37, 705-720 (2019)

23. M. Guillaud, D. Slock, R. Knopp, A practical method for wireless channel reciprocity exploitation through relative calibration. In: Proceedings of the 8th International Symposium on Signal Processing and Its Applications: 28-31 June; Sydney (2005)

24. G. Stüber, Principles of Mobile Communication (Springer, New York, 2017)

25. S. Kay, Fundamentals of Statistical Signal Processing: Estimation Theory (Prentice Hall, Upper Saddle River, 1993).

26. T. Fawcett, An introduction to ROC analysis. Pattern Recogn. Lett. 27, 861-874 (2006)

27. A. Oppenheim, G. Verghese, Signals, Systems and Inference (Pearson, New York, 2015).

28. K. Baddour, T. Willink, Improved estimation of the Ricean K-factor from I/Q fading channel samples. IEEE Trans. Wirel. Commun. 7, 5051-5057 (2008)

29. E. Aboutanios, B. Mulgrew, Iterative frequency estimation by interpolation on Fourier coefficients. IEEE Trans. Signal Process. 53, 1237-1242 (2005)

30. A. Goldsmith, Wireless Communications (Cambridge University Press, Cambridge, 2005).

\section{Publisher's Note}

Springer Nature remains neutral with regard to jurisdictional claims in published maps and institutional affiliations.

\section{Submit your manuscript to a SpringerOpen ${ }^{\odot}$ journal and benefit from:}

- Convenient online submission

- Rigorous peer review

- Open access: articles freely available online

- High visibility within the field

Retaining the copyright to your article

Submit your next manuscript at $\gg$ springeropen.com 